\title{
Absolute LIBS stratigraphy with Optical Coherence Tomography
}

\author{
Ewa A. Kwiatkowska ${ }^{*}$, Jan Marczak ${ }^{\mathrm{b}}$, Roman Ostrowski ${ }^{\mathrm{b}}$, Wojciech Skrzeczanowski ${ }^{\mathrm{b}}$, Marcin \\ Sylwestrzak $^{\mathrm{a}}$, Magdalena Iwanicka ${ }^{\mathrm{c}}$, and Piotr Targowski ${ }^{\mathrm{a}}$ \\ ${ }^{a}$ Institute of Physics, Nicolaus Copernicus University, ul. Grudziądzka 5, 87100 Toruń, Poland \\ ${ }^{\mathrm{b}}$ Institute of Optoelectronics, Military University of Technology, ul. Kaliskiego 2, \\ 00-908 Warszawa, Poland \\ ${ }^{c}$ Institute for the Study, Restoration and Conservation of Cultural Heritage, Nicolaus Copernicus \\ University, ul. Gagarina 7, 87-100 Torun, Poland
}

\begin{abstract}
In this contribution preliminary studies on the application of Optical Coherence Tomography (OCT) to absolute depth calibration of Laser Induced Breakdown Spectroscopy (LIBS) data in application to revealing stratigraphy of easel paintings are presented. The procedure of in-situ monitoring of LIBS by means of OCT is described. Numerical method developed for precise extraction of the depth of the LIBS ablation crater is explained. Results obtained with model paintings are discussed.
\end{abstract}

Keywords: LIBS, laser-induced breakdown spectroscopy, OCT, optical coherence tomography, stratigraphy, depth profiling, micro-invasive examination of art

\section{INTRODUCTION}

Laser Induced Breakdown Spectroscopy (LIBS) [1] is well established technique for analyzing the chemical composition of various objects of art. Briefly: plasma generated from the investigated material by short laser pulse is analyzed spectrally for presence of specific atom and ion lines to gain information of elemental composition of the object. Since present detection techniques based on CCD detectors with image intensifiers are very sensitive, the necessary volume of material analyzed is very little. Therefore the ablation crater can be very small, usually with diameter of the fraction of a millimetre. This justifies classification of the LIBS method as micro-invasive and usually acceptable from the point of view of conservation ethics. Consequently, this technique presently became popular for identification of various materials from pigments in polychromy and pottery to resolving composition of metal alloys [2-4]. However, if the item under investigation is built of layers composed of different chemical elements, results obtained via this simple way may not be conclusive. In such a case a known remedy is so called LIBS profile analysis or stratigraphy, when consecutive laser pulses are applied to the same place [3-5]. Their energy is adjusted at the low level to ensure ablation of a thin layer of the material (in range from 0.1 to $10 \mu \mathrm{m}$ ) in one shot. In such a case collected spectra consecutively reveal the composition of strata within the object. The disadvantage of this approach is in lack of absolute information on the depth from which certain spectrum is collected and the determination of layer thickness is not possible. Furthermore, even the relation of thicknesses of compared layers cannot be yielded by simple counting of numbers of laser pulses, because the ablation rate of different strata may vary significantly.

This drawback may be overcome by means of Optical Coherence Tomography (OCT) - a new, fast-growing technique for non-contact and non-destructive structural imaging of semi-transparent objects. The OCT method employs the interferometry of light of high spatial but low temporal coherence to measure locations of scattering elements of internal structure of the object examined. In result a cross-sectional view - a tomogram - of the object is obtained. If this optical sectioning is repeated many times in adjacent parallel locations, a 3D structure may be recovered. In addition, by numerical recognition of the air-object interface, a precise profile of its surface may be obtained. Since 2004 OCT has been applied for examination of various objects of cultural heritage [6-9]. In this contribution a novel utilisation of OCT

*ewka@fizyka.umk.pl; phone +48 56611 3214; fax +48 566225397 
is presented. When used in conjunction with LIBS, it can serve not only as a tool aiding proper selection of the place of testing with LIBS, but also, what is even more important, it may be used for monitoring the process of the LIBS crater etching. Specifically, in case of LIBS depth profiling, the depth of ablation crater may be directly measured after each laser pulse and thus the stratigraphic profile may be scaled in depth absolutely.

\section{METHODOLOGY}

\subsection{The LIBS system}

To generate plasma gentle pulses of fourth harmonic of Nd-YAG laser (ReNOVALaser 5, $\lambda=266 \mathrm{~nm}$, pulse energy $=$ $6.5 \mathrm{~mJ}$, pulse duration $\sim 10 \mathrm{~ns}$ ) were used. Laser radiation was focused on the sample with a lens and ablation craters of about $300 \mu \mathrm{m}$ in diameter were gradually formed. To reach the prime layer of the sample painting usually from 10 to 15 pulses were necessary.

Echelle Spectra Analyzer ESA 4000 from LLA Instruments GmbH was used to analyze radiation from plasma. The instrument was equipped with a MCP (gated microchannel plate) image intensifier and an iCCD sensor array (Kodak KAF-1001). The spectral range of the Echelle spectrometer was $200 \mathrm{~nm}$ to $780 \mathrm{~nm}$ with a linear dispersion varying from $5 \mathrm{pm} /$ pixel at $200 \mathrm{~nm}$ to $19 \mathrm{pm} /$ pixel at $780 \mathrm{~nm}$. Theoretical spectral resolution $\lambda / \Delta \lambda$ was about 20000 . Spectral data were collected for $10 \mu \mathrm{s}$ with $2 \mu \mathrm{s}$ delay after laser pulse to avoid background radiation from free electrons generated during ablation process. To analyze spectra ESAWIN 4.1.4 software with spectral data base ROI Service were used.

\subsection{The OCT instrument}

Cross-sectional images of the sample and their surface profiles were collected with a high-resolution Spectral OCT tomograph build in the Institute of Physics, Nicolaus Copernicus University especially for examination of cultural heritage artefacts. Detailed description of this instrument is given elsewhere in this volume [10]. It is capable of creating cross-sectional images (tomograms) of objects weakly absorbing light with axial resolution of $4 \mu \mathrm{m}$. The instrumental limit of in-depth imaging is about $2 \mathrm{~mm}$ and thus sufficient for examination of thin structures like paint layers. The transversal resolution is about $30 \mu \mathrm{m}$. Tomograms (Fig. 2b, Fig. 3) are shown in false colours scale: high scattering centres are shown as light spots or in warm colours. Low scattering media remains dark or blue. Cross-section images collected in adjacent positions may be combined into volume data, usually seen as a cloud of points. Picture in figure 3 is complemented with the image of the sample's surface. This surface is automatically generated from the OCT data and shown with elevation coded by the lightness of its colour (HSL colour model), essentially as described elsewhere [11].

\subsection{The sample}
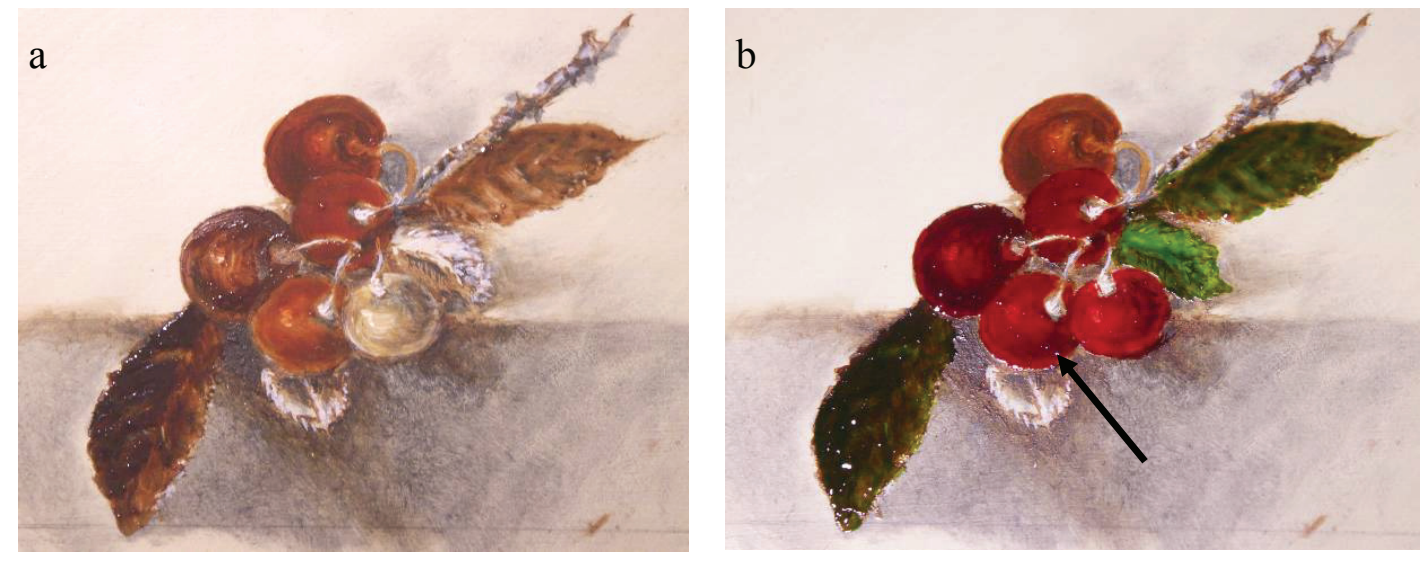

Fig. 1.a: sample panel during preparation (after application of underdrawing and underpainting layers); b: sample panel after application of finishing glaze layer; arrow indicates place of LIBS data acquisition

\section{E.A. Kwiatkowska et al. "Absolute LIBS stratigraphy with Optical Coherence Tomography”, Proc of SPIE 7391, p. 7391-14}

Copyright 2009 Society of Photo-Optical Instrumentation Engineers. One print or electronic copy may be made for personal use only. Systematic reproduction and distribution, duplication of any material in this paper for a fee or for commercial purposes, or modification of the content of the paper are prohibited. 
Test sample panel imitating traditional painting techniques was prepared specially for this experiment. It consisted of primed wooden support, underdrawn with deoiled mixture of burnt umber, gold ochre and Payne's grey (Fig. 1a). In order to create different possible visual and structural effects, a subsequent treatment of the painted cherries was then varied by means of using different colour and amount of layers in the underpainting. The area in which the experiment was finally conducted (arrow in Fig. 1b) had been underpainted with a single layer of burnt sienna mixed with oil-resin painting medium and after some time finished with one layer of glaze (synthetic carmine alizarine with medium) Fig. 2. Whereas it was not anticipated that the organic compounds embedded in synthetic lakes could be identified with the LIBS analysis, diversification of spectra obtained from the two different layers of the model sample was expected.

a

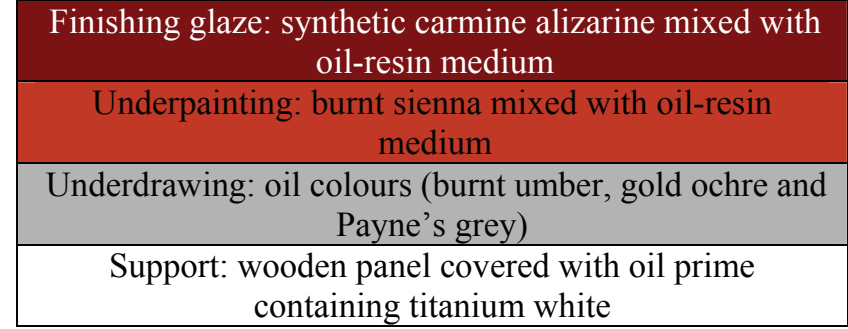

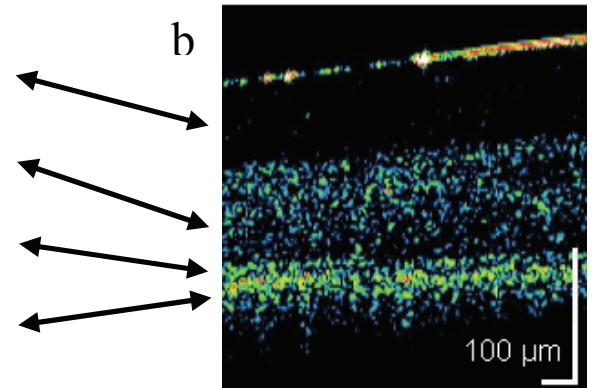

Fig. 2. a: sample stratigraphy in place of LIBS data acquisition (arrow in Fig. 2); b: OCT tomogram of the same place collected before acquisition of LIBS data

\subsection{Experiment procedure and data processing}

After each pulse volume OCT data were collected. From this data the profile of the ablation crater was recovered and thus its depth precisely determined. Independently, the analysis of LIBS spectra provided information on chemical composition of the plasma. Finally, the absolute profile of the sample was composed from both data.

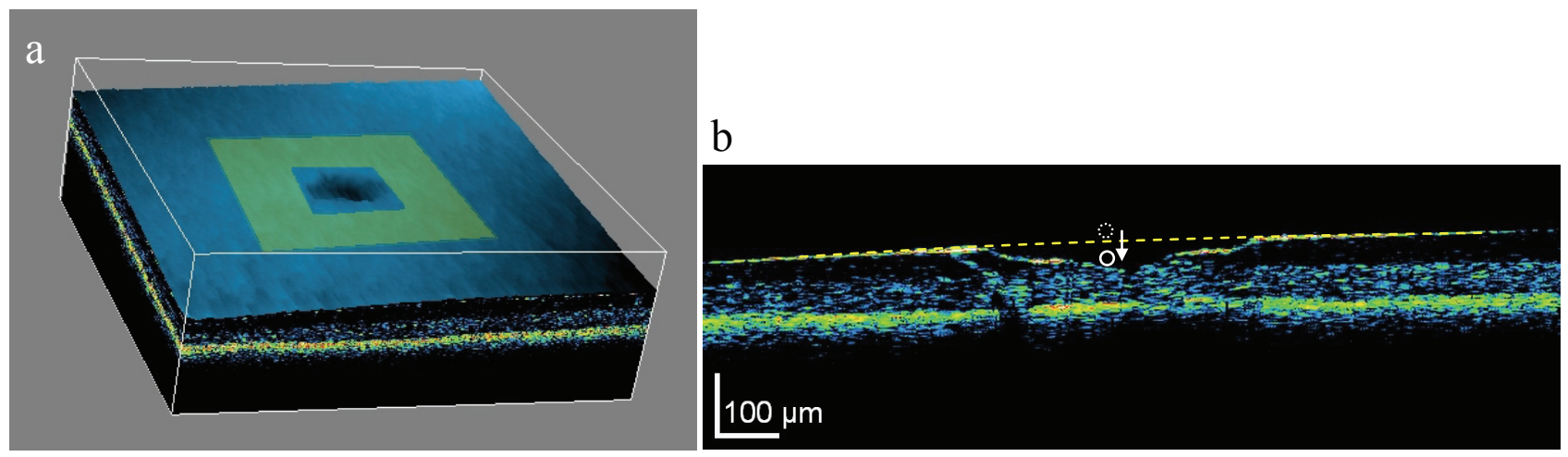

Fig. 3. a: OCT 3D tomogram from the sample from Fig. 1 after LIBS analysis - ablation crater is visible in the centre. OCT image is not corrected for refraction: cage dimensions are $3.2 \times 3.2 \times 0.8 \mathrm{~mm}$. Sample surface shown in blue. Reference area for crater depth determination is shown in yellow; $b$ : an example OCT tomogram (corrected for refraction) used for crater depth determination, dashed yellow line represent the reference surface interpolated from the fragment of sample surface marked in yellow in Fig. 3a - see text for details

To precisely determine the depth of ablation crater a following procedure was adopted. Firstly the surface of the sample was recovered from the OCT data, essentially by searching for the first edge from the top in the OCT image. This surface is shown in blue in Fig. 3a. Since the surface's recognition in an area of the ablation crater was not univocal, the selected surface data (yellow area in Fig. 3a) was used to interpolate the reference plane only. Then from this plane a virtual ball was dropped down (Fig. 3b) until it touched the material of the sample. This way small discontinuities in the scattering

\section{E.A. Kwiatkowska et al. "Absolute LIBS stratigraphy with Optical Coherence Tomography”, Proc of SPIE 7391, p. 7391-14}

Copyright 2009 Society of Photo-Optical Instrumentation Engineers. One print or electronic copy may be made for personal use only. Systematic reproduction and distribution, duplication of any material in this paper for a fee or for commercial purposes, or modification of the content of the paper are prohibited. 
signal (caused by strong speckle effect, for instance) didn't influence the result. The maximum of dropping distances in the perimeter of the whole crater was taken as a measure of the crater's depth. This procedure was repeated for various ball diameters. The diameter $(18 \mu \mathrm{m})$ used for final determination of the crater depth was chosen from the range where the depth recovered was practically independent of the ball diameter.

It is worthwhile to note that for this procedure neither refraction correction nor even sample transparency for inspecting OCT beam is necessary. This is because only the signal from scattering at the sample's surface is analysed for this purpose. It broadens potential range of applications of this technique to any for which the LIBS analysis is applicable. However, if the paint layers within the sample are transparent enough to permit OCT imaging, these cross-sectional images are very helpful in interpretation of LIBS data (see Fig. 4b-f for examples). In such a case refraction correction of the OCT images is strongly recommended.

\section{RESULTS}

An exemplary results obtained from OCT data for the model sample introduced in figures 1 and 2 are presented in Fig. 4a. An error of crater's depth determination is estimated to $\pm 4 \mu \mathrm{m}$. After $11^{\text {th }}$ pulse it became apparent from the OCT cross-section that the crater reached the wooden support of the painting and the experiment was terminated. In the figure three depth ranges (marked with dashed lines in the figure and colours corresponding to Fig. 2a) of different ablation rates are clearly seen: first until $40 \mu \mathrm{m}$ with rate of $11.2 \pm 0.3 \mu \mathrm{m} /$ pulse, then from $40 \mu \mathrm{m}$ to $70 \mu \mathrm{m}$ with rate of $5.5 \pm 0.2$ $\mu \mathrm{m} /$ pulse and finally the strata with rate of $3.2 \pm 0.2 \mu \mathrm{m} /$ pulse. Difference in ablation speed is evident.

a

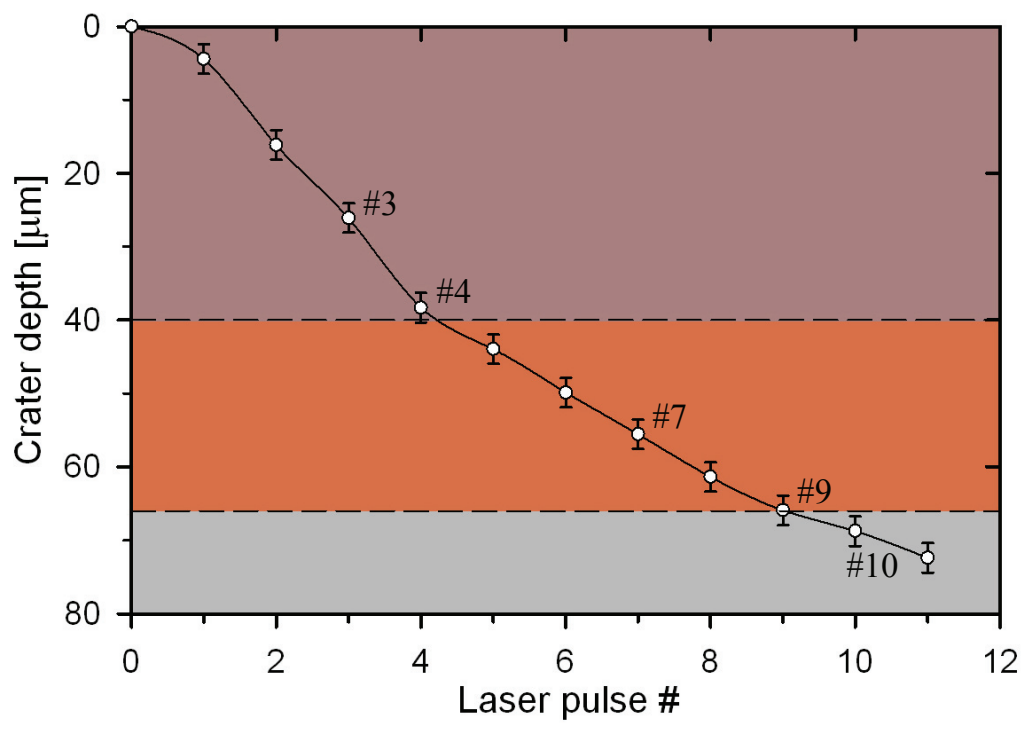

b

\#3

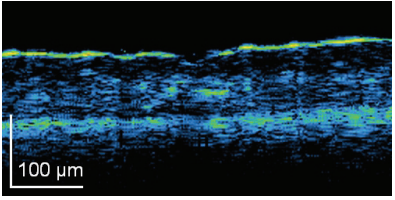

\#4

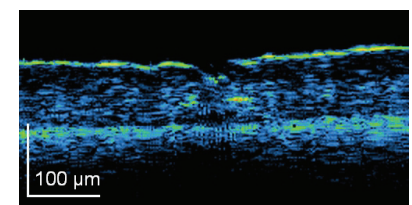

d

\#7

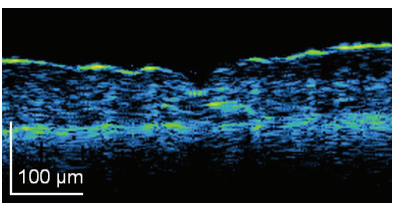

e

\#9

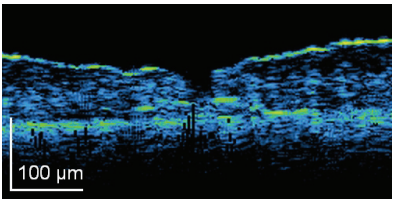

\#10

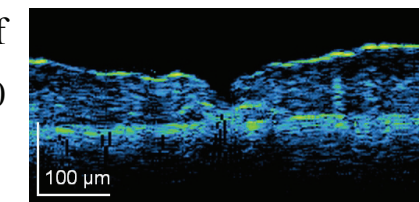

Fig. 4. a: in-depth calibration curve obtained from OCT data for LIBS experiment with sample shown in Figs 1-3; b-f: refraction-corrected OCT tomograms after $3^{\text {rd }}, 4^{\text {th }}, 7^{\text {th }}, 9^{\text {th }}$, and $10^{\text {th }}$ pulse respectively 
Comparison with the OCT cross-sectional images of the ablation crater (Fig 4. b-f) shows excellent correlation between these ranges of ablation rates and depths of the ablation crater in respect to the sample structure: first strata which extends until $40 \mu \mathrm{m}$ beneath the sample's surface is thus a finishing glaze layer (Fig. 3) and ends with pulse \#4 (Fig. 4c), then between $40 \mu \mathrm{m}$ and $70 \mu \mathrm{m}$ lays the underpainting, and finally below $70 \mu \mathrm{m}$ the underdrawing and support layers are located.

This kind of rough identification is very helpful in the LIBS analysis. In case of pigment identification obtained spectra are very complicated. Moreover, if a stratified sample is examined additional difficulty comes from the lack of sharp boundaries between layers - their chemical composition changes gradually due to intended or diffusive mixing. Quantitative analysis of LIBS spectra might be easier with increased counts number. However, potential damage to painting layer caused by higher energy of laser pulse makes it a drawback. Nevertheless, calibration curve from Fig. 4 permits associating these spectral signals with specific depths and layers within the paint. In Fig. 5 examples of LIBS spectra from different layers are shown. Some intense lines of elements present in spectra are marked.

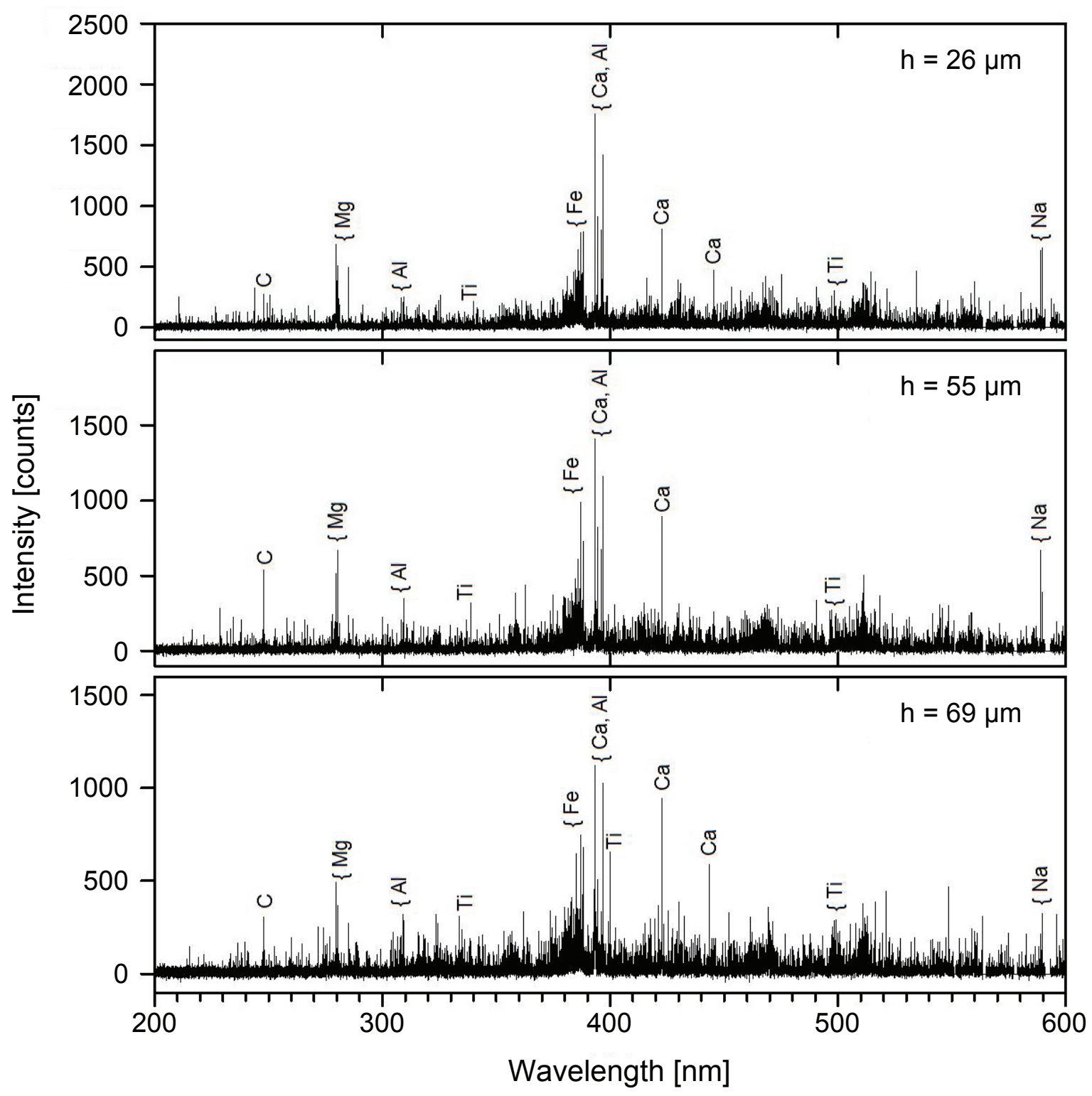

Fig. 5. Exemplary LIBS spectra obtained for depths identified with OCT examination 
However, quantitative analysis of composition of these strata is limited by varying intensities of plasma fluorescence for subsequent laser shots. To overcome this drawback a ratio of intensities of chosen lines may be analysed. To obtain such relative concentration profile reliable over all paint depth special considerations may be given to proper choice spectral lines. First of all they should have significant intensity in all spectra collected and must not overlap with any other lines from elements present in any strata of the sample. Additionally it is desired that both transitions should have theirs upper states of similar energy to minimize the influence of plasma temperature on the spectral lines intensity. Taking all this into account titanium line at $\lambda=336,121 \mathrm{~nm}$ and iron line at $\lambda=385,990 \mathrm{~nm}$ were chosen. Their intensities are sufficient, they are not overlapping with other lines present in our data and energies of upper states of their transitions are $3.7 \mathrm{i}$ $3.2 \mathrm{eV}$ respectively. Obtained result is shown in Fig. 6. Again, dashed lines indicate the same three in-depth ranges corresponding to three strata in the sample's structure. The minimum of Ti/Fe ratio is observed for depth from 40 to $55 \mu \mathrm{m}$ which corresponds to burnt sienna underpainting layer rich in iron. Interpretation of the Ti/Fe ratio increase in the upper strata (glaze) is not univocal - separate examination of titanium and iron lines shows some significant intensities from both titanium and iron. However, the latter is lower then in the next layer, containing sienna. Third, underdrawing and prime layer is rich in titanium and it is evident from data shown in the figure.

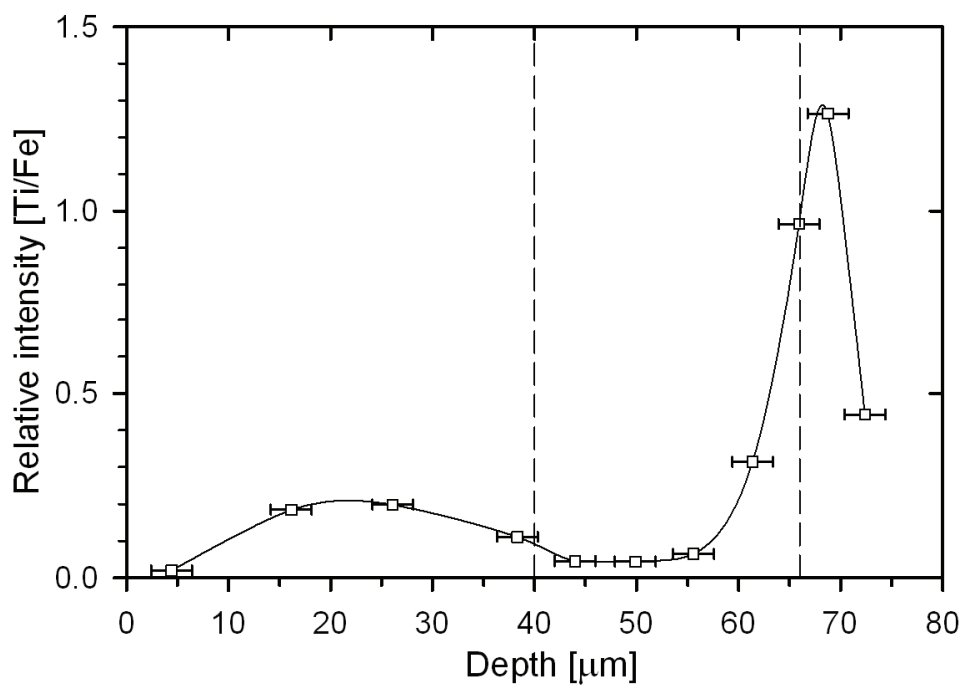

Fig. 6. An example of absolute depth profile analysis by examining of relative intensity of titanium line at $\lambda=336,121 \mathrm{~nm}$ versus iron line at $\lambda=385,991 \mathrm{~nm}$ as a function of depth calibrated from the OCT data

\section{CONCLUSIONS}

Optical Coherence Tomography method may be used for absolute in-depth calibration of LIBS stratigraphy data. To do so, only information on sample surface must be recovered and thus, in principle, sample doesn't have to be transparent for IR light used in OCT analysis. This means that this functionality of the OCT technique has broader application then usual cross-sectioning. Nevertheless, if cross-sectional images of the sample in place of examination are available, this additional information is very helpful in interpretation of LIBS data. This is especially important in demanded case of depth profiling of pigmented layers in paintings.

\section{ACKNOWLEDGEMENT}

This work was supported by Polish Government Research Grants through the years 2008-2011. EK, MS, and MI gratefully acknowledge additional support from European Social Fund and Polish Government within Integrated Regional Development Operational Programme, Action 2.6, by project "Stypendia dla doktorantów 2008/2009 ZPORR" of Kuyavian-Pomeranian Voivodeship.

\section{E.A. Kwiatkowska et al. "Absolute LIBS stratigraphy with Optical Coherence Tomography”, Proc of SPIE 7391, p. 7391-14}

Copyright 2009 Society of Photo-Optical Instrumentation Engineers. One print or electronic copy may be made for personal use only. Systematic reproduction and distribution, duplication of any material in this paper for a fee or for commercial purposes, or modification of the content of the paper are prohibited. 


\section{REFERENCES}

[1] Yueh F., Singh J. P., and Zhang H., Laser-induced Breakdown Spectroscopy, [Elemental Analysis, Encyclopaedia of Analytical Chemistry, R.A. Meyers (Ed.)] John Wiley \& Sons Ltd, Chichester, 2066-2087 (2000).

[2] Melessanaki K., Mateo M., Ferrence S.C., Betancourt P.P., Anglos D., The application of LIBS for the analysis of archaeological ceramic and metal artifacts, Applied Surface Science 1997-198, 156-163 (2002).

[3] Fotakis C., Anglos D., Zafiropulos V., Georgiou S., Tornari V., [Lasers in the Preservation of Cultural Heritage, Principles and Applications] CRC Press, Boca Raton, 53-87 (2006).

[4] Mendes N.F.C., Osticioli I., Striova J., Sansonetti A. Becucci M., Castellucci E., Versatile pulsed laser setup for depth profiling analysis of multilayered samples in the field of cultural heritage, Journal of Molecular Structure 924-926, 420-426 (2009).

[5] Anderson D.R, McLeod C.W, English T., Trevor Smith A., Depth Profile Studies Using Laser-Induced Plasma Emission Spectrometry, Applied Spectroscopy 49(6), 691-701 (1995).

[6] Targowski P., Rouba B., Wojtkowski M., Kowalczyk A., Application of optical coherence tomography to nondestructive examination of museum objects, Studies in Conservation 49, 107-114 (2004).

[7] Liang H., Peric B., Hughes M., Podoleanu A.G., Spring M., Roehrs S., Optical Coherence Tomography in archaeological and conservation science - a new emerging field, Proc. SPIE 7139, 713915-1 - 713915-9 (2008).

[8] Yang M.-L., Winkler A.M., Barton J.K., Vandiver P.B., Using optical coherence tomography to examine the subsurface morphology of Chinese glazes, Archeometry 50, DOI: 10.1111/j.1475-4754.2008.00451.x (2008).

[9] complete list of papers on application of OCT to examination of art: www.oct4art.eu

[10] Targowski P., Ostrowski R., Marczak J., Sylwestrzak M., Kwiatkowska E.A., Picosecond laser ablation system with process control by Optical Coherence Tomography, Proc SPIE 7391, 739115 (2009).

[11] Sylwestrzak M., Kwiatkowska E.A., Karaszkiewicz P., Iwanicka M., Targowski P., Application of graphically oriented programming to imaging of structure deterioration of historic glass by Optical Coherence Tomography Proc SPIE 7391, 739109-1 (2009). 